\title{
Liquidez y rentabilidad en un colegio privado
}

\section{Liquidity and profitability in a private school}

\section{Liquidez e lucratividade em escola particular}

\section{Roselin Aurora Laura Villanueva \\ 1auroralaur@gmail.com \\ https://orcid.org/0000-0002-5041-950x}

Universidad Peruana Unión, Perú
Noemí Roxana Phala Turpo

noemiphala07@gmail.com

iD https://orcid.org/0000-0002-8105-1605

Universidad Peruana Unión, Perú

Recibido: 18 de junio de 2021

Aceptado: 30 de diciembre de 2021

\section{Resumen}

En el mundo empresarial, la liquidez y la rentabilidad son dos indicadores financieros, permiten evaluar las inversiones en una entidad; las expectativas de optimización de sus valores permiten plantear una posible relación entre estas; el presente estudio buscó analizar el grado de relación existente entre la liquidez y la rentabilidad. El estudio es correlacional; se realizó el análisis de los estados financieros, del periodo 2017 - 2019; los resultados dejan la evidencian de que no existe una relación significativa $(\leq 0.05)$ entre las ratios de la liquidez y la rentabilidad; según el Rho de Spearman, la escala de la relación se da en un nivel bajo. Se concluye que, durante el periodo 2017-2019, la razón corriente, la prueba ácida, la razón líquida y el capital de trabajo no presentaron una relación significativa con la rentabilidad económica y financiera en el centro educativo investigado.

Palabras clave: Liquidez, rentabilidad, ratios de liquidez, ratios de rentabilidad, razones financieras. 


\section{Abstract}

In the business world, liquidity and profitability are two financial indicators, they allow evaluating investments in an entity; the optimization expectations of their values allow to propose a possible relationship between them; The present study sought to analyze the degree of relationship between liquidity and profitability. The study is correlational; The analysis of the financial statements was carried out for the period 2017 - 2019; The results show that there is no significant relationship $(\leq 0.05)$ between the liquidity and profitability ratios; according to Spearman's Rho, the scale of the relationship is at a low level. It is concluded that, during the period 2017-2019, the current ratio, the acid test, the liquid ratio and the working capital did not present a significant relationship with the economic and financial profitability in the studied educational center.

Keywords: Liquidity, profitability, liquidity ratios, profitability ratios, financial ratios.

\section{Resumo}

No mundo dos negócios, liquidez e rentabilidade são dois indicadores financeiros, pois permitem avaliar os investimentos de uma entidade; as expectativas de otimização de seus valores permitem propor uma possível relação entre eles; O presente estudo buscou analisar o grau de relação entre liquidez e lucratividade. O estudo é correlacional; A análise das demonstrações financeiras foi realizada para o período 2017 - 2019; Os resultados mostram que não existe relação significativa $(\leq 0,05)$ entre os índices de liquidez e rentabilidade; de acordo com Rho de Spearman, a escala do relacionamento está em um nível baixo. Conclui-se que, no período 2017-2019, o índice corrente, o acid test, o índice de liquidez e o capital de giro não apresentaram relação significativa com a rentabilidade econômico-financeira do centro educacional estudado.

Palavras-chave: Liquidez, lucratividade, índices de liquidez, índices de rentabilidade, índices financeiros.

\section{Introducción}

Bajo la premisa empresarial de que la mayor motivación de toda entidad es el crecimiento económico, se prioriza el adecuado manejo de la liquidez y los márgenes adecuados de rentabilidad: principales indicadores financieros; sobre esto, García (2014) 
explica que se debe a que la optimización de estos permite cumplir las obligaciones inmediatas y el sostenimiento de la entidad.

Para entender su interacción es preciso definirlas teóricamente; en ese sentido, Daza (2016) menciona que la rentabilidad es un concepto económico, financiero y social; en el campo empresarial, refiere la facultad de una empresa para generar un excedente a partir de una inversión efectuada; se puede asegurar que es más un resultado de una actividad productiva, debido a que siempre aparece en la parte final del proceso. Según Chávez y Contreras (2018), existen dos tipos de rentabilidad: la económica y la financiera.

En la definición que presentan Cuadros et al. (2016), se señala que la rentabilidad es un concepto económico que expresa el grado particular de una empresa, para cumplir sus propias obligaciones corrientes a corto plazo; esto implica la capacidad puntual de conversión de los activos en disponible, por lo que también recibe el nombre de solvencia. Por su parte, Paz y Taza (2017) expresan que, si bien la liquidez actúa como indicador financiero, para poder medirla se debe utilizar las ratios que la componen, a lo que según Ugarte et al. (2017) se presentan como Capital de trabajo, Razón corriente, Prueba ácida y Razón líquida.

La búsqueda alineada del inversionista sobre estos dos referentes, permiten suponer una asociación teórica; los estudios evaluados sobre estos indicadores resaltan a Gutiérrez y Tapia (2016), Tizón (2017), Berrenechea (2016), Chávez y Contreras (2018) y Pomalaza (2016); aunque los resultados no ameritan concordancia, la teoría sostiene que existe una relación inversa entre la rentabilidad y la liquidez; es decir, las inversiones con una mayor liquidez obtendrán una rentabilidad menor; tal como lo explica Mejía et al. (2020), cuanto más difícil le resulte a un inversor poder convertir un activo en dinero líquido, mayor será la rentabilidad que exija a dicho activo; claro esta que esta afirmación está sujeta a las intenciones de la inversión y es precisamente en este punto donde los resultados de los estudios comienzan a diferir.

García (2014) expone los posibles escenarios de estos indicadores dentro de una empresa, los cuales son esencialmente cuatro; el primero es cuando la rentabilidad y la liquidez son categorizadas de buena; es decir, está consolidada y denota el desarrollo económico de la empresa, debido a la generación de utilidades, lo que activa el flujo de efectivo y resulta una posición óptima para toda empresa. El segundo escenario es la insuficiencia de liquidez, el cual resulta un indicador de crecimiento financiero para la 
empresa, lo que se traduce en un escaso flujo en las operaciones y, por lo tanto, el cumplimiento de los compromisos de la empresa se encuentra postergado.

El tercer escenario es que la empresa no cuente ni con liquidez ni rentabilidad óptima; lo cual demuestra un estado crítico de la situación financiera de la entidad, la misma que podría buscar financiamiento externo. El último escenario es donde no se percibe rentabilidad, pero si liquidez; porque la empresa aunque no perciba ganancias, logra convertir sus recursos en activos para mantener sus operaciones, originando la disminución de su patrimonio hasta el punto en que pueda revertir su condición.

En forma resumida, los problemas financieros de corto plazo se encuentran asociados con la liquidez, debido al requerimiento de los compromisos inmediatos y que imposibiliten el ejercicio de las actividades empresariales. En cambio, los problemas financieros a largo plazo se relacionan con la rentabilidad, debido a que su optimización representa una garantía futura de la liquidez; por tal motivo, se hace imprescindible el uso de estrategias que logren maximizarla; un ejemplo podría ser la reducción de los costos o el incremento del volumen de las ventas. Se puede decir que frente a un problema financiero, la prioridad de solución la tiene la liquidez, mientras la rentabilidad amerita un estudio detallado y un planeamiento más elaborado para revertir su afección.

En conclusión, en el mundo empresarial siempre se busca la supervivencia, permanencia y crecimiento de las empresas, en un mercado altamente competitivo, que exige al empresario estar atento a los cambios y las necesidades que se presenten, con el fin de garantizar el adecuado funcionamiento y la autonomía financiera que se necesita, para sobrevivir en este medio; pero erróneamente se tiene la concepción de buscar la liquidez y la rentabilidad como factores independiente, sin analizar que está íntimamente relacionados, lógicamente de un acertado equilibrio entre ambas, precisamente es, en este punto, donde está el mayor desafío para las empresas.

En cuanto a la iniciativa de la investigación, esta presenta un interés particular en el rubro de la educación, debido a que, como actividad de servicio, se encuentra con una demanda creciente a nivel mundial; en ese sentido, la investigación encuentra su justificación en el desarrollo de la educación en el país; Avellaneda (2016) considera que lo estudios en materia financiera para este rubro son escasos, a pesar de la importancia que amerita el manejo de este tipo de entidades, por lo que se espera aportar a los intereses nacionales. 
La realidad del contexto objeto de estudio, obedece a la observación del manejo eficiente de la contabilidad; aunque su sostenimiento es evidencia de un buen prestigio y ejercicio de la actividad educativa, se hace necesario investigar su crecimiento en base a los indicadores de rentabilidad y liquidez que ha venido presentando durante los últimos tres (3) años; esto podría favorecer la realización de proyecciones en la toma de decisiones, con respecto a esto. En tal sentido, Pomalaza (2016) sostiene que el análisis de indicadores resulta una herramienta financiera que permite a los negocios ser más competitivos y exitosos, concretamente en materia de inversiones, razón por la cual el objetivo de este estudio es analizar el grado de relación que existe entre las ratios de liquidez y la rentabilidad en un colegio privado de la ciudad de Huancayo.

\section{Metodología}

El tipo de investigación es correlacional, de diseño no experimental y corte transversal (Hernández et al., 2014). En cuanto a la delimitación espacial, ésta ubica al colegio Privado Adventista Huancayo, teniendo como objeto de estudio a los estados financieros de la institución.

La población está constituida por todos los estados financieros del Colegio Adventista Huancayo, desde su fundación en 1956, lo que hace un total de 63 balances anuales, excluyendo el que corresponde al año 2020, por no haberse concluido todavía.

En ese sentido, el interés de los investigadores y la accesibilidad de la información por parte de la institución, se presenta un criterio de exclusión a los años anteriores al 2017; bajo esta consideración, se tiene que mediante un muestreo no probabilístico por conveniencia, se utilizaron los balances contables anuales del colegio Adventista Huancayo y que corresponden a los años 2017, 2018 y 2019.

La técnica de investigación utilizada fue el análisis documentario, una forma de investigación técnica, que hace uso de una secuencia de operaciones intelectuales para describir y representar información previamente documentada de forma unificada y sistemática, lo que, según Dulzaides y Molina (2004), comprende una extracción científico-informativa que busca reflejar la realidad original.

El procesamiento de los datos partió de la recolección por medio de una técnica e instrumento establecido para la realidad investigativa; se procedió luego a un cálculo de las ratios financieras, el mismo que fue sometido a un análisis estadístico de correlación. 
El instrumento fue la ficha de análisis (hoja de cálculo), definida por Bernardo et al. (2019) como una estructura diseñada intencionalmente, para recopilar y organizar información en conjunto; en este caso, la investigación pretende recoger los datos cuantitativos que representan las ratios provenientes de los balances contables del colegio Adventista Huancayo.

Las ratios calculadas comprenden las variables liquidez y rentabilidad y fueron efectuados en el programa Microsoft Excel; posteriormente, se introducen como data estadística en el programa MINITAB 19, con un nivel de significancia de 0.05.

\section{Resultados y discusión}

El tratamiento estadístico analizó de manera inicial, la comprobación del supuesto de la normalidad para todas las ratios de liquidez y rentabilidad (ver tabla 1). Para esto se eligió la prueba de normalidad Shapiro Wilk, considerada una prueba potente para tamaños de muestra $\geq 30 \mathrm{y} \leq 50$ (Flores et al., 2019).

Tabla 1

\begin{tabular}{ll}
\hline Variable & Valor sig. \\
\hline Razón corriente & 0.231 \\
Prueba ácida & 0.229 \\
Razón Líquida & 0.229 \\
Capital de trabajo & 0.140 \\
Rentabilidad financiera & $<0.0010$ \\
Rentabilidad Económica & $<0.0010$ \\
\hline
\end{tabular}

Prueba de Shapiro wilk

Nota: Los resultados del test de Normalidad sugieren la elección de una prueba no paramétrica, en este caso se eligió la prueba Rho de Spearman (Ver Tabla 2). Las ratios de la liquidez como razón corriente, prueba ácida, razón líquida y capital de trabajo, fueron analizados en combinaciones con las ratios de rentabilidad económica y financiera.

La tabla 2 detalla el valor obtenido para cada combinación, por lo que se observa también que ninguno de los valores es significativo $(<0.05)$, por lo que se puede decir que existe evidencia estadística suficiente, para afirmar que no existe relación significativa entre las ratios de liquidez y las ratios de la rentabilidad.

En cuanto al Rho de Spearman, este difiere entre la combinación del tipo de rentabilidad; es así que los ratios de la liquidez como Razón corriente, prueba ácida y Razón líquida (Rho de Spearman $=0.240)$, capital de trabajo $($ Rho de Spearman=0.196) 
y la rentabilidad financiera, presentan coeficientes que indican una tendencia positiva de nivel bajo; en cambio, las combinaciones de los mismos ratios de liquidez: Razón corriente, Prueba ácida, Razón líquida (Rho de Spearman =0.082) y la rentabilidad económica presentan coeficientes de tendencia positiva de nivel muy bajo y en el caso del capital de trabajo (Rho de Spearman= - 0.023) una tendencia negativa o comportamiento inverso también de nivel muy bajo.

Tabla 2

Prueba de correlación de Spearman entre ratios de liquidez y rentabilidad.

\begin{tabular}{lll}
\hline Relación & $\begin{array}{l}\text { Rho de } \\
\text { Spearman }\end{array}$ & Valor P \\
\hline Razón corriente $\leftrightarrow$ Rentabilidad financiera & 0.240 & 0.158 \\
Prueba ácida $\leftrightarrow$ Rentabilidad financiera & 0.240 & 0.158 \\
Razón líquida $\leftrightarrow$ Rentabilidad financiera & 0.240 & 0.158 \\
Capital de trabajo $\leftrightarrow$ Rentabilidad financiera & 0.196 & 0.253 \\
Razón corriente $\leftrightarrow$ Rentabilidad económica & 0.082 & 0.634 \\
Prueba ácida $\leftrightarrow$ Rentabilidad económica & 0.082 & 0.634 \\
Razón Líquida $\leftrightarrow$ Rentabilidad económica & 0.082 & 0.634 \\
Capital de trabajo $\leftrightarrow$ Rentabilidad económica & -0.023 & 0.894
\end{tabular}

Nota: Un estudio correlacional similar de Gutiérrez y Tapia (2016), quienes sostienen que la razón corriente, la razón ácida y líquida no tienen relación con la rentabilidad económica, pero si con la rentabilidad financiera. La diferencia de los resultados pareciera explicarlo Berrenechea (2016), quien afirma que la mayoría de las empresas del sector educativo privado, presentan problemas de liquidez y solvencia; lo curioso de los resultados es que la liquidez y la rentabilidad no siempre se encuentran directamente proporcionales, argumentando que esto se debe a múltiples factores de influencia.

Por su parte, Chávez y Contreras (2018), en un estudio ex-post-facto en la misma red educativa, encuentran que existe relación positiva entre el manejo de las políticas de cobranza con la liquidez, ocurriendo de manera negativa para el caso de la morosidad y 
sostienen que reestructurando las políticas de crédito se mejora la competitividad en el mercado educativo.

En cuanto a la tendencia positiva, Vásquez et al. (2020) encuentran que la rentabilidad y la liquidez tienen una relación positiva y significativa, pero recalcan que los resultados son según el marco definido.

\section{Conclusiones}

La liquidez y la rentabilidad del colegio dentro del periodo analizado, no se relacionan significativamente ( $\mathrm{p}$ valor $<0.05$ ); se puede afirmar que los resultados que aseguran una relación entre la liquidez y la rentabilidad, se encuentran condicionados al contexto empresarial y dependerá de los objetivos con los que se realicen las inversiones.

A pesar de los resultados no significativos, la tendencia de la relación Liquidez, en sus ratios Razón corriente, prueba ácida y razón liquida, con la Rentabilidad económica es positiva, muy baja; no obstante, el capital de trabajo y la rentabilidad económica son de tendencia inversa; por otro lado, la tendencia de la relación de las ratios de liquidez y la Rentabilidad financiera es positiva y muy baja.

De acuerdo con el estudio efectuado, se pudo observar que los márgenes de rentabilidad y los índices de liquidez se encuentran en estado adecuado, lo que evidencia una buena gestión financiera; asimismo, se observa que aunque la organización percibe aportes considerables por parte del colegio, también permite un margen de inversión interna, por lo que se recomienda buscar el crecimiento institucional a través de reinversiones a largo plazo.

\section{Referencias bibliográficas}

Avellaneda, R. (2016). Rentabilidad del colegio particular San Joaquín y Santa Ana de Cutervo, Cajamarca en el periodo 2014. Universidad Señor de Sipán.

Bernardo, C., Carbajal, Y. y Contreras, V. (2019). Metodología de la investigación: manual del estudiante. Universidad San Martín de Porres. Recuperado de https://www.usmp.edu.pe/estudiosgenerales/pdf/2019I/MANUALES/II\%20CICLO /METODOLOGIA\%20DE\%20LA\%20INVESTIGACION.pdf

Berrenechea, I. (2016). Economic and financial analysis of education sector. Universidad de Cantabria. 
Chávez, J. y Contreras, M. (2018). Gestión de las cuentas por cobrar y la situación económica y financiera de la Asociación Educativa Adventista Peruana del Norte, periodo 2013-2017. Universidad Peruana Unión.

Cuadros, R., Rojas, C. y Vargas, H. (2016). Gestión de Cuentas por cobrar y su incidencia en la liquidez de la empresa Apple Glass Peruana SAC 2015. Universidad Peruana las Américas.

Daza, J. (2016). Crecimiento y rentabilidad empresarial en el sector industrial brasileño. Contaduría y administración: (1)61, 266 - 282. Recuperado de http://www.scielo.org.mx/pdf/cya/v61n2/0186-1042-cya-61-02-00266.pdf

Dulzaides, M. y Molina, A. (2004). Análisis documental y de información: dos componentes de un mismo proceso. ACIMED 12 (2). Recuperado de http://eprints.rclis.org/5013/1/analisis.pdf

Flores, P., Muñoz, L. y Sánchez, T. (2019). Estudio de Pruebas de normalidad usando distribuciones desconocidas con distintos niveles de no normalidad. Perfiles, 1 (21) https://www.researchgate.net/publication/332863672 ESTUDIO DE POTENCIA DE_PRUEBAS_DE_NORMALIDAD_USANDO_DISTRIBUCIONES_DESCO NOCIDAS_CON_DISTINTOS_NIVELES_DE_NO_NORMALIDAD

Gutiérrez, A. y Tapia, J. (2016). Relación entre liquidez y rentabilidad de las empresas del sector industrial que cotizan en la Bolsa de Valores de Lima, 2005-2014. Universidad Peruana Unión, Perú. Recuperado de https://repositorio.upeu.edu.pe/bitstream/handle/UPEU/466/Joel_Tesis bachiller_2 016.pdf? sequence $=1 \&$ is Allowed $=\mathrm{y}$

García, Y. (2014). Liquidez y rentabilidad como factor determinante en el éxito de las empresas. Universidad de San Buenaventura. Colombia. Recuperado de http://bibliotecadigital.usb.edu.co/bitstream/10819/2235/1/Liquidez\%20y\%20rentabilidad \%20como\%20factor Lizeth\%20Garc\%C3\%ADa\%20Restrepo e 2014.pdf

Gutiérrez, J. y Jhonatan, T. (2016). Relación entre liquidez y rentabilidad de las empresas del sector industrial que cotizan en la Bolsa de Valores de Lima, 2005-2014. Universidad Peruana Unión.

Hernández, R., Fernández, C. y Baptista, M. (2014). Metodología de la investigación. https://doi.org/- ISBN 978-92-75-32913-9 


\section{Liquidez y rentabilidad en un colegio privado}

Mejía, K., Sicheri, L. y Nolazco, F. (2020). La liquidez en los procesos económicos de una empresa de decoración de interiores, Lima 2019. Rev. Espíritu Emprendedor TES, 4(1) 1- 12. Recuperado de https://doi.org/10.33970/eetes.v4.n1.2020.178

Pomalaza, G. (2016). La administración de la liquidez y su efecto en el resultado de las empresas arrendadoras de centros comerciales de lima metropolitana. Universidad San Martín de Porres.

Paz, E. y Taza, Y. (2017). La gestión financiera en la liquidez de la empresa Yossev eirl del distrito del Callao durante el periodo 2012. Universidad de Ciencias y Humanidades.

Tizón, P. (2017). Relación entre capital de trabajo y rentabilidad de las empresas industriales que cotizan en la Bolsa de Valores de Lima, 2010 - 2015.Universidad Peruana Unión. Perú. Recuperado de https://repositorio.upeu.edu.pe/handle/UPEU/859

Ugarte, E., León, J. y Parra, G. (2017). La trampa de liquidez, historia y tendencias de investigación. Revista Problemas de Desarrollo: 48 (190), 165-188. Recuperado de https://www.jstor.org/stable/90012739?seq=1

Vázquez, X., Rech, I., Miranda, J. y Tavares, M. (2020). Convergencias entre la rentabilidad y la liquidez en el sector del agronegocio. Cuadernos de Contabilidad 18(45). Recuperado de http://www.scielo.org.co/pdf/cuco/v18n45/0123-1472-cuco18-45-00152.pdf 